\section{INVISIBILIDADE DA PREVENÇÃO DE CÂNCER EM MULHERES COM DOENÇA MENTAL: UM RELATO DE EXPERIÊNCIA}

\section{INVISIBILITY OF CANCER PREVENTION IN WOMEN WITH MENTAL ILLNESS: AN EXPERIENCE REPORT}

Jaine Kareny da Silva ${ }^{1}$ / Joice Mara Amorim Messias, ${ }^{2, *}$

\section{INTRODUÇÃO}

O Ministério da Saúde (MS) organizou o Programa de Assistência Integral à Saúde da Mulher (PAISM) em 1984, incluindo ações educativas, preventivas, diagnóstico, tratamento e recuperação na assistência ginecológica, prénatal, parto e puerpério, climatério, câncer de colo de útero e de mama, e outras necessidades identificadas a partir do perfil populacional das mulheres (BRASIL, 2004).

Apesar disso, este cuidado integral não tem sido efetivado na prática, o que torna necessário mudar o modelo atual de atenção à saúde das mulheres, como por exemplo, para aquelas com transtorno mental, cuja assistência tem sido centrado nas doenças psíquicas. O objetivo de mudança do modelo assistencial biologista-fragmentado é oferecer atendimento mais humano, holístico e eficaz, de forma que a integralidade e as questões de gênero sejam ligadas na formação dos profissionais que atendem os diversos grupos populacionais, podendo assim, intervir com ações direcionadas a essa realidade, considerando as suas particularidades. Esta necessidade de vincular as questões de gênero, socioeconômicas e culturais é reforçada pela Política Nacional de Atenção Integral a Saúde da Mulher (BRASIL, 2004).

Neste cenário, as mulheres com doença mental também apresentam outros problemas de saúde que necessitam de atenção integral, como a prevenção de cânceres a partir da periodicidade da avaliação ginecológica. Tal necessidade se justifica pelos dados apresentados pelo Instituto Nacional do Câncer (INCA), em que, somente no ano de 2018 aconteceram 59.700 casos novos de câncer de mama feminino, 6.600 casos novos de câncer de colo de útero, e 16.370 casos novos de câncer de colo do útero. A mortalidade em 2017 foi de 16,724 óbitos por câncer de mama, 6.385 óbitos por câncer de colo de útero e 3.879 óbitos por câncer de ovário (INCA, 2019).

\section{RESUMO}

O objetivo deste estudo é relatar a invisibilidade da saúde integral à mulher com doença psíquica. Trata-se de um relato de experiência realizado após observação de enfermeiras e estudantes de enfermagem de uma instituição particular de ensino superior, no período de março à maio de 2018, durante um estágio supervisionado que ocorreu de segunda a sexta- feira, em turnos alternados no CAPS II de uma cidade da região oeste do interior baiano. A discussão realizada com a equipe de saúde mental demonstra a dificuldade destes profissionais em atenderem ao princípio de integralidade e o funcionamento do sistema de Referência e Contrarreferência do Sistema Único de Saúde para as mulheres com doença mental afetadas por câncer, apesar de seu conhecimento sobre a sua importância. Portanto, é necessário que esta temática e suas nuanças sejam debatidas nos espaços de formação estudantil e profissional, com vistas a garantir melhor resolutividade na saúde dessas mulheres, considerando-se as questões de gênero e a sua condição de adoecimento mental.

Palavras-chave: Saúde da mulher. Saúde mental. Neoplasias da mama. Neoplasias de Mama. Neoplasia uterina. Integralidade em saúde.

\section{ABSTRACT}

This study is intended to report the invisibility of integral health for women with mental illness. This is an experience report performed after observation of nurses and nursing students from a private higher education institution, during the period from March to May 2018, while attending a supervised internship that took place from Monday to Friday, in alternate shifts at the CAPS II located in a city of the western region of the hinterland of Bahia. The discussion held with the mental health team shows the difficulty of these professionals in meeting the principle of integrality and the operation of the Reference and Counter-Reference system of the Unified Health System for women with mental illness affected by cancer, despite their knowledge about the importance of both topics. Therefore, it is necessary that this theme and its details be debated in the spaces for student and professional training, in order to ensure better resolution in the health of these women, taking into account gender issues and their condition of mental illness.

Keywords: Women's Health. Mental Health. Breast Neoplasms. Uterine Neoplasms. Integrality in Health.

Submetido em: 08 de nov. 2019

Aceito em: 14 de fev. 2020

${ }^{1}$ Universidade do Estado da Bahia - UNEB, Guanambi, Bahia - Brasil.

${ }^{2}$ Universidade do Estado da Bahia - UNEB, Serrinha, Bahia - Brasil

*E-mail para correspondência: joiceamorim.enfermagem@ hotmail.com 
Neste contexto de prevenção e tratamento do câncer, a saúde mental feminina sempre foi estigmatizada (ZANELLO; SILVA, 2012). Há, portanto, uma limitação na rede assistencial à saúde, pois as unidades especializadas não conseguem atender efetivamente estas mulheres de maneira integral, limitando-se ao acolhimento das demandas relacionadas ao sofrimento mental (BARBOSA; DIMENSTEIN; LEITE, 2014).

Como essas mulheres com doença mental apresentam uma dificuldade de gerenciar o cuidado à saúde, é importante conhecer sobre o funcionamento da atenção integral nos serviços de saúde pública, incluindo a saúde ginecológica preventiva. Por tanto, o objetivo deste estudo é relatar a invisibilidade da saúde integral à mulher com doença psíquica. Por conseguinte, espera-se despertar a importância da prevenção de câncer de colo de útero e de mama em todas as mulheres nos espaços de atenção psicossocial em saúde.

Dessa forma, esse estudo tornase relevante, pois suscita discussões acerca das lacunas enunciativas sobre este tema e provocam inquietações na vida prática de profissionais, o que leva a reflexão do modo como ocorre esta problemática no cenário nacional e quais potencialidades podem de traçadas para mudança deste paradigma de fragmentação assistencial.

\section{MATERIAIS E MÉTODOS}

Trata-se de um relato de experiência que é parte de um estudo de campo, de cunho exploratório e abordagem qualitativa. Lakatos e Marconi (2010) descrevem que a pesquisa de campo é empregada com objetivo de conhecer e obter informações sobre determinado problema, a partir da formulação de questões investigativas, o que aumenta a familiaridade do pesquisador com o ambiente, fato ou fenômeno. Esta relação, entre pesquisador e objeto de estudo, favorece a compreensão do fenômeno social (RICHARDSON et al., 2011).

A observação foi realizada por enfermeiras e estudantes de enfermagem de uma instituição particular de ensino superior, no período de março à maio de 2018, durante um estágio supervisionado que ocorreu de segunda a sexta- feira, em turnos alternados. O local de estudo foi o CAPS II de, que atende diversos usuários e, entre eles, mulheres que também são atendidas nos demais serviços da rede de atenção à saúde. A referida unidade localiza-se em uma cidade da região oeste do interior baiano, cuja população é estimada em 123.741 habitantes (IBGE, 2019).

Durante as atividades de estágio supervisionado no CASP II, duas professoras e 03 alunos evidenciaram uma usuária de 32 anos com dificuldade locomotora, queixas importantes de dor pélvica e que nunca havia realizado coleta do exame ginecológico. Como a usuária se socializava efetivamente com toda equipe visitante $\mathrm{e}$ estava em companhia de uma familiar (mãe), foi possível realizar uma anamnese satisfatória com ambas. Por meio de uma conversa informal, a mãe da usuária relatou metrorragia há alguns dias e que o resultado de uma ultrassonografia revelou a presença de uma 'massinha'. Contudo, como a dor havia cessado, a mãe não comunicou estes fatos à equipe de saúde mental. Todas as informações cedidas pela familiar foram confirmadas pela usuária.

Diante desta realidade, a equipe universitária visitante levantou indagações sobre como a discussão da saúde física, em especial a prevenção de cânceres, era abordada no âmbito da saúde mental. Esta discussão incluiu a reflexão sobre a integralidade assistencial e o funcionamento do sistema de referência e contrarreferência, uma vez que algumas usuárias daquele serviço também tiveram gestação, aborto, histórico de violência sexual e física. Apesar disso, a preocupação daquela unidade era direcionada para a realização de consultas em saúde mental, renovação de receitas e oficinas de artes, mas não se atentavam para outros fatores que podem acometer a mulher em todo seu ciclo de vida.

Para viabilizar a discussão, questionou-se à equipe da unidade de saúde mental se: I) essas mulheres relatavam a realização do exame preventivo do colo uterino e das mamas nas unidades de atenção básica e eram orientadas sobre a importância desta prevenção no CAPS; II) eram informadas sobre a necessidade de reportarem alteração corporal à equipe da unidade saúde mental ou outra pessoa de sua confiança; ou III) traziam a família/cuidadores/responsáveis para eles também serem orientados.

Para a análise e discussão do tema proposto, utilizou-se artigos oriundos da bases de dados da Biblioteca Virtual de Saúde (BVS), que integra várias bases de dados referenciais como LILACS (Literatura LatinoAmericana e do Caribe em Ciências da Saúde), e publicações do MS relacionadas ao tema.

\section{RESULTADOS E DISCUSSÃO}

A integralidade é um dos princípios do Sistema Único de Saúde (SUS) preconizados na Lei 8.080/90, que considera as pessoas como um todo, contemplando todas as suas necessidades, incluindo ações de promo- 
ção da saúde, prevenção de doenças, tratamento e reabilitação. Esse princípio garante que haja a articulação com outras políticas públicas, para assegurar atuação intersetorial entre as diferentes áreas que possam refletir na saúde e qualidade de vida das mulheres com doença mental (BRASIL, 1990).

Apesar deste princípio ser preconizado pelo SUS, os profissionais de saúde do referido serviço, responderam que não tinham como fornecer suporte assistencial à saúde física e que este apoio era uma responsabilidade da Estratégia de Saúde da Família (ESF) da área adscrita. Todavia, apesar do uso da tecnologia leve, como a educação em saúde horizontalizada, ser um método simples, eficaz e disponível na atenção básica do município, não deve limitar-se à esta unidade de atenção primária à saúde.

É preciso que os profissionais trabalhem juntos para integrar as suas práticas de maneira intersetorial dentro da rede de atenção à saúde. As unidades de saúde mental devem expandir suas práticas para além da realização de consultas grupais com profissionais da saúde mental, renovação de receitas e oficinas. Assim, a práxis do cuidado não deve ser pensada e trabalhada em partes, mas como o funcionamento destas partes afeta todo o sistema de saúde física e mental.

Como já exposto, essas mulheres em sofrimento mental apresentam dificuldade de cuidar do próprio corpo, e relatar sinais e sintomas de diferentes doenças. Diante disto, a Organização Mundial da Saúde (OMS) ressalta a importância de ações para a detecção e diagnóstico precoce, principalmente para o rastreamento em população assintomática, com objetivo de identificar lesões ou alterações sugestivas de câncer de colo de útero e mama e en- caminhá-las para investigação e tratamento necessário. Assim, a incidência e a mortalidade por câncer do colo do útero e de mama podem ser reduzidas com programas organizados e articulados de rastreamento (OMS, 2007).

Outros estudos também apontam que há uma dificuldade dos profissionais de saúde em manterem ações integrativas. Inserir as especialidades nos programas de saúde se fez importante, porém essa precisa ser trabalhada de forma integrada de modo a oferecer ações continuadas aos usuários. Conforme Sousa et al. (2012), a dificuldade de executar ações de integralidade no serviço de saúde acontece quando há a fragmentação do trabalho desenvolvido nas unidades básicas, comprometendo assim os princípios da atenção primária à saúde; outras causas para tais problemas estão no fato de muitas unidades promoverem atendimentos ambulatoriais sem adequá-las às necessidades específicas da população atendida.

Reforçando tal ideia, Franco et al., (1999) diz que a superespecialização causada pela fragmentação do trabalho leva o profissional de saúde à ficar alheio ao seu objeto de trabalho. Nesse sentido, os trabalhadores isolados do principal objetivo do trabalho em saúde - o paciente/usuário - não terão compromisso com o resultado agrupado das ações que deveriam ser realizadas em benefício do paciente.

No que tange ao nãoatendimento às necessidades das mulheres, tal falha repercute nas experiências vividas com a sociedade. Com seus direitos violados, elas sofrem com as dificuldades de construir vínculos com o serviço de saúde o que compromete a qualidade do serviço e prejudica a saúde da mulher, uma vez que essa corre o risco de mesmo com algum sintoma importante, não queixar aos profissionais por não possuir laços de confiança (OLIVEIRA; FONSECA, 2015).

Dados do MS mostram que existem cerca de seis milhões de mulheres entre 35 a 49 anos que nunca realizaram o exame citopatológico do colo do útero. Esta é a idade de maior incidência do câncer do colo do útero e a realização do exame preventivo é a principal estratégia para detectar lesões cervicais e fazer o diagnóstico precoce da doença (INCA, 2019). Neste sentido, as ações educativas e compartilhamento de informações na rede de atenção à saúde são importantes para essas mulheres com doença mental, pois aumentam as chances de tratamento e prevenção dessa doença.

Não foi percebido durante a observação realizada no estágio supervisionado, o funcionamento do sistema de referência e contrarreferência informal (e.g. ligações telefônica) ou formalmente (e.g. registro no prontuário) para essas usuárias, o que contradiz o princípio da integralidade. Os próprios profissionais reforçavam em seus discursos que "a prevenção de câncer de colo de útero e mama já existe na ESF e não há como 'entrar' na área dos outros colegas", o que demonstra a resistência destes trabalhadores, que poderiam utilizar o vínculo criado com essas usuárias para melhorar à assistencial integral em sua saúde.

O sistema de Referência e Contrarreferência é uma estratégia de organização do SUS para garantir acesso das pessoas aos serviços de saúde. Assim, o usuário atendido em uma determinada unidade básica - se houver necessidade - deverá ser encaminhado para um serviço de maior complexidade e receber o atendimento que precisa, o que se constitui na referência. Logo após o atendimento desta 
necessidade, o usuário será encaminhado à unidade de origem para que a continuidade do atendimento seja feita, o que representa a contrarreferência (BRASIL, 2011).

Nota-se, portanto, que a continuidade do serviço é garantida pelo SUS, mas os profissionais do serviço em estudo distorcem o seu significado e, por conseguinte, fragmentam a assistência à saúde para essas usuárias, o que contraria a própria definição de saúde estabelecida pela OMS ao descrever que "a saúde é o completo bemestar físico, mental e social, e não a mera ausência de doenças" (OMS, 1947).

\section{CONCLUSÃo}

Os resultados oriundos das discussões mostram a dificuldade dos profissionais dos serviços de saúde mental em perceber essas usuárias como um ser humano holístico, e por isso limitam as suas ações no foco da doença mental, negligenciando assim, o princípio de integralidade e o sistema de referência e contrarreferência do SUS. Portanto, é necessário que estes temas sejam discutidos no processo de formação durante a graduação e na formação continuada em serviço para que haja uma melhor resolutividade na saúde dessas mulheres, considerandose as questões de gênero e a sua condição de adoecimento mental.

\section{REFERÊNCIAS}

BARBOSA, Laís Barreto; DIMENSTEIN, Magda; LEITE, Jáder Ferreira. Mulheres, violência e atenção em saúde mental: questões para (re) pensar o acolhimento no cotidiano dos serviços. Avanços em Psicologia Latinoamericana, Bogotá, v. 32, n. 2, p. 309320, May/Aug, 2014.
BRASIL. Ministério da Saúde. Lei n ${ }^{\circ}$ 8.080, de 19 de setembro de 1990. Dispõe sobre as condições para promoção, proteção e recuperação da saúde, a organização e o funcionamento dos serviços correspondentes e dá outras providências. Diário Oficial da União: Seção 1, Brasília, DF, 20/09/1990. Disponível em: http://www.planalto.gov.br/ccivil_03/1 eis/18080.htm. Acessado em 03 de outubro de 2019 às 23:45min.

BRASIL. Ministério da Saúde. Secretaria de Atenção à Saúde. Departamento de Ações Programáticas Estratégicas. Política nacional de atenção integral à saúde da mulher: princípios e diretrizes / Ministério da Saúde, Secretaria de Atenção à Saúde, Departamento de Ações Programáticas Estratégicas. Brasília: DF, 2004.

BRASIL. Conselho Nacional de Secretários da Saúde-CONASS. Atenção Primária e promoção da saúde: Coleção Progestores - Para entender a gestão do SUS. Brasília: CONASS, 2011.

FRANCO, Túlio Batista; BUENO, Wanderlei Silva; MERHY, Emerson Elias. O acolhimento e os processos de trabalho em saúde: o caso de Betim, Minas Gerais, Brasil. Cadernos de Saúde Pública, Rio de Janeiro, v. 15, n. 2, p. 345-353, abr-jun, 1999.

\section{IBGE - INSTITUTO BRASILEIRO} DE GEOGRAFIA E ESTATÍSTICA.

População: Barreiras, Bahia. Disponível em:

https://cidades.ibge.gov.br/brasil/ba/ba rreiras; acessado em 04 de outubro de 2019 às 19:49min.

INCA - INSTITUTO NACIONAL DO CANCER. Câncer. Brasil, 2019.

Disponível em:

https://www.inca.gov.br/numeros-decancer; acessado em 02 de outubro de 2019 às 21:00h.

LAKATOS, Eva Maria; MARCONI, Marina de Andrade. Fundamentos de metodologia científica: Técnicas de pesquisa. 7 ed. São Paulo: Atlas, 2010.
OLIVEIRA, Rebeca Nunes Guedes de; FONSECA, Rosa Maria Godoy Serpa da. Necessidades em saúde: a interface entre o discurso de profissionais de saúde e mulheres vitimizadas. Rev. Latino-Am. Enfermagem, São Paulo, v. 23, n. 2, p. 299-306, 2015.

OMS - ORGANIZAÇÃO MUNDIAL DE SAÚDE. Controle de Câncer.

Conhecimento em ação. Guia da OMS para programas eficazes. OMS, 2007. Disponível em:

<www.who.int/cancer/modules/Preven tion\%20Module.pdf; acessado em 05 de outubro de 2019.

OMS - ORGANIZAÇÃO MUNDIAL DA SAÚDE. Constituição da Organização Mundial da Saúde. Documentos básicos, 1947. Disponível em: http://www.who.int/governance/eb/wh o_constitution_sp.pdf.

RICHARDSON, Roberto Jarry et al. Pesquisa Social, Métodos e Técnicas. 3 ed. São Paulo: Editora Atlas, 2011.

SOUZA, Márcio Costa de et al. Integralidade na atenção à saúde: um olhar da Equipe de Saúde da Família sobre a fisioterapia. O Mundo da Saúde, São Paulo, v. 36, n. 3, p. 452-460, 2012.

ZANELLO, Valeska; SILVA, René Marc Costa e. Saúde Mental, Gênero e Violência Estrutu-

ral. Bioética, Brasília, v. 20, n. 2, p. 267-279, 2012. 